\title{
Research on Multi - time Scale Modeling and Simulation Technology for AC / DC Hybrid Distribution Network
}

\author{
Siqin $\mathrm{Li}^{1, \mathrm{a}}$, Hairong Luo ${ }^{1, \mathrm{~b}, *}$, Yongning Huang ${ }^{1, \mathrm{c}}$ and Cunxi Bai ${ }^{1, \mathrm{~d}}$ \\ ${ }^{1}$ Electric Power Research Institute of State Grid NingXia Electric Power Company, \\ Jinfeng District, Yinchuan City, NingXia Autonomous Region, China. \\ b13209598368@163.com
}

Keywords: AC / DC hybrid distribution network, control Strategy, Multi-time scale

Abstract: AC / DC hybrid distribution networks need to be simulated at different time scales in specific scenes. In this paper, based on the different particle size of the model, a multi-time scale simulation model of AC / DC hybrid distribution network is established on the basis of establishing the mathematical model and control strategy of the converter component of AC / DC hybrid distribution network. Based on the IEEE 33-node example, Matlab / Simulink is used to simulate the running process of AC / DC hybrid distribution network at each time scale, and the accuracy of the simulation results is compared with each time scale. The experimental results show that the simulation waveform of AC / DC hybrid distribution network model is more accurate.

\section{Introduction}

With the rapid development of the power electronic device and flexible AC transmission technology, the status of DC distribution technology in power distribution network is more and more important, the AC / DC hybrid power distribution network as the transition network accepted DC power distribution technology, has become a hot topic at present. Therefore, it is very important to analyze and simulate the AC / DC hybrid distribution network converter and its control strategy.

Researchers at home and abroad have done a lot of research on the modeling of converters and their control strategies in AC / DC hybrid distribution networks. [1] [3] on the various types of converter were studied, the establishment of converter mathematical model and carried out a simulation analysis. In the literature [4] [7], the control strategy of the converter is discussed, and the control strategy modeling of PQ, V / f and Droop is studied emphatically. In the literature [8] [10], the photovoltaic arrays are modeled and the output characteristics of PV arrays under normal conditions and partial occlusion are analyzed by combining the changing trend of PV-I-U characteristics under different conditions.

Based on the mathematical model of AC / DC hybrid distribution network, this paper has carried on the multi-scale modeling to the commutation link, has carried on the simulation to the circulation mirror and its control strategy in the same scene at different scales, has carried on the comparison to the result, And then verify the feasibility of the multi-scale model. 

Network

\subsection{Converter link structure}

$\mathrm{AC}$ and DC hybrid distribution network commutation links are divided into rectifier links and inverter links in two parts, as shown in Figure 1. Among them, the AC side to the DC side of the process of power transmission: rectifier through a certain control strategy to control the AC power into $\mathrm{DC}$, from the $\mathrm{AC}$ side to the $\mathrm{DC}$ side of the power supply. The process of transmitting power from the $\mathrm{DC}$ side to the $\mathrm{AC}$ side is that the inverter is controlled by a certain control strategy to convert the direct current into alternating current and to transmit power from the $\mathrm{DC}$ side to the $\mathrm{AC}$ side.

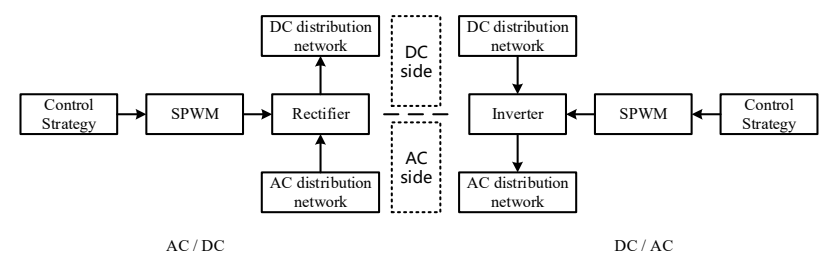

Figure 1 Schematic Diagram of AC / DC hybrid distribution network commutation link.

The mathematical model of the components of the circulation is established below to further model it.

\subsection{Mathematical model of converter}

\subsubsection{AC / DC converter}

A typical three-phase full-bridge AC / DC converter based on VSC technology is shown in Figure 2, consisting mainly of IGBTs and filters (filter resistors and filter reactors).

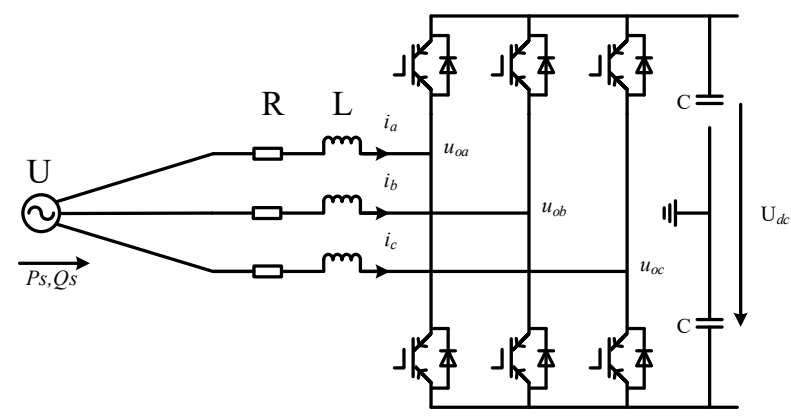

Figure 2 Topology of AC / DC Converter.

In Figure 2, $\mathrm{U}$ is the AC side of the distribution voltage, $i_{a} 、 i_{b} 、 i_{c}$ for the AC side three-phase current, $u_{o a} 、 u_{o b} 、 u_{o c}$ for the AC side three-phase voltage, $\mathrm{U}_{d c}$ for the converter DC side voltage, $\mathrm{R}$ for the filter Resistance, $\mathrm{L}$ is the filter equivalent inductance.

The differential equation of AC / DC converter based on Kirchhoffs voltage law (KVL): 


$$
\left\{\begin{array}{l}
u_{o a}=u_{s a}-R i_{a}-L \frac{\mathrm{d} i_{a}}{\mathrm{~d} t} \\
u_{o b}=u_{s b}-R i_{b}-L \frac{\mathrm{d} i_{b}}{\mathrm{~d} t} \\
u_{o c}=u_{s c}-R i_{c}-L \frac{\mathrm{d} i_{c}}{\mathrm{~d} t}
\end{array}\right.
$$

Formula (1) through the park transformation, the three-phase static coordinate system into two-phase $d-q$ rotating coordinate system under the mathematical model equation:

$$
L \frac{\mathrm{d}}{\mathrm{d} t}\left[\begin{array}{l}
i_{d} \\
i_{q}
\end{array}\right]=\left[\begin{array}{cc}
0 & -\omega L \\
\omega L & 0
\end{array}\right]\left[\begin{array}{l}
i_{d} \\
i_{q}
\end{array}\right]+\left[\begin{array}{l}
u_{s d} \\
u_{s q}
\end{array}\right]-\frac{M U_{d c}}{2}\left[\begin{array}{c}
\cos \delta \\
\sin \delta
\end{array}\right]
$$

In the formula, $i_{d}$ and $i_{q}$ are the current $\mathrm{d}$ and the q-axis component, $\omega$ is the synchronization angular frequency, $u_{s d}$ and $u_{s q}$ are the power supply side voltage $\mathrm{d}$ and the q-axis component, $\mathrm{M}$ is the modulation ratio, and $\delta$ is the firing angle.

Formula (2) through the coordinate transformation of the rotating coordinate system under the power of the formula:

$$
\left\{\begin{array}{l}
P_{\mathrm{s}}=\frac{3}{2}\left(u_{\mathrm{sd}} i_{\mathrm{d}}+u_{\mathrm{sq}} i_{\mathrm{q}}\right) \\
Q_{\mathrm{s}}=\frac{3}{2}\left(u_{\mathrm{sd}} i_{\mathrm{q}}-u_{\mathrm{sq}} i_{\mathrm{d}}\right)
\end{array}\right.
$$

Formula (3), when the AC side voltage is a standard symmetrical three-phase sine wave, u_sq is zero, it can be simplified as:

$$
\left\{\begin{array}{l}
P_{\mathrm{s}}=\frac{3}{2} u_{\mathrm{sd}} i_{\mathrm{d}} \\
Q_{\mathrm{s}}=\frac{3}{2} u_{\mathrm{sd}} i_{\mathrm{q}}
\end{array}\right.
$$

The control strategies of AC / DC converters can be designed according to equations (3) and (4).

\subsection{2. $\mathrm{DC} / \mathrm{AC}$ converter}

A typical three-phase full-bridge DC / AC converter based on VSC technology is shown in Figure 3, consisting mainly of IGBTs and filters (filter capacitors and filter reactors).

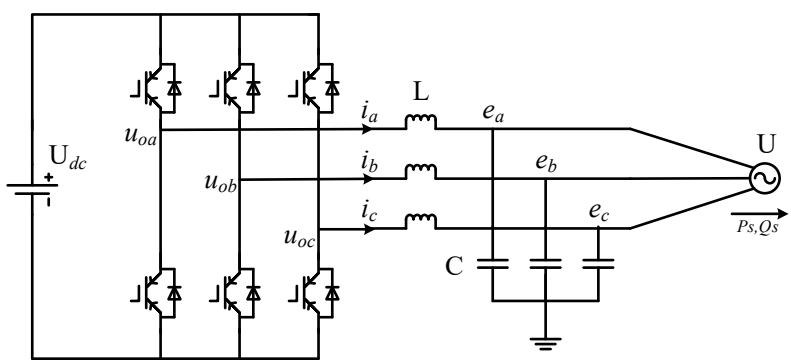

Figure 3 Topology of DC / AC Converter.

Figure 3, $\mathrm{U}_{d c}$ for the converter DC side voltage, $u_{o a}, u_{o b}, u_{o c}$ for the filter before the exvariable side of the three-phase voltage, $i_{a} 、 i_{b}, i_{c}$ for the AC side three-phase current, $\mathrm{L}$ for the filter equivalent inductance, $\mathrm{C}$ for the filter Equivalent capacitance, $e_{a}, e_{b}, e_{c}$ is, $\mathrm{U}$ is the filtered AC side voltage.

The differential equation of DC / AC converter based on Kirchhoffs voltage law (KVL): 


$$
\left\{\begin{array}{l}
u_{o a}=e_{a}+L \frac{\mathrm{d} i_{a}}{\mathrm{~d} t} \\
u_{o b}=e_{b}+L \frac{\mathrm{d} i_{b}}{\mathrm{~d} t} \\
u_{o c}=e_{c}+L \frac{\mathrm{d} i_{c}}{\mathrm{~d} t}
\end{array}\right.
$$

Formula (1) through the park transformation, the three-phase static coordinate system into two-phase d-q rotating coordinate system under the mathematical model equation:

$$
L \frac{\mathrm{d}}{\mathrm{d} t}\left[\begin{array}{l}
i_{d} \\
i_{q}
\end{array}\right]=\left[\begin{array}{cc}
0 & \omega L \\
-\omega L & 0
\end{array}\right]\left[\begin{array}{l}
i_{d} \\
i_{q}
\end{array}\right]+\left[\begin{array}{l}
u_{d} \\
u_{q}
\end{array}\right]-\left[\begin{array}{l}
e_{d} \\
e_{q}
\end{array}\right]
$$

Formula (6) through the coordinate transformation of the rotating coordinate system under the power calculation formula:

$$
\left\{\begin{array}{l}
P_{\mathrm{s}}=\frac{3}{2}\left(u_{\mathrm{d}} i_{\mathrm{d}}+u_{\mathrm{q}} i_{\mathrm{q}}\right) \\
Q_{\mathrm{s}}=\frac{3}{2}\left(e_{\mathrm{q}} i_{\mathrm{d}}-e_{\mathrm{d}} i_{\mathrm{q}}\right)
\end{array}\right.
$$

Formula (7), when the grid side voltage is a standard symmetrical three-phase sine wave, $e_{\mathrm{q}}$ is zero, it can be simplified as:

$$
\left\{\begin{array}{c}
P_{\mathrm{s}}=\frac{3}{2} e_{\mathrm{d}} i_{\mathrm{d}} \\
Q_{\mathrm{s}}=-\frac{3}{2} e_{\mathrm{d}} i_{\mathrm{q}}
\end{array}\right.
$$

The control strategies of DC / AC converters can be designed according to equations (7) and (8).

\subsection{Converter control strategy}

The control strategy mainly includes constant power control strategy (PQ control strategy), constant voltage constant frequency control strategy (V / f control strategy), droop control strategy (Droop control strategy) and DC voltage control and other control strategies. According to the converter and distributed power supply different, and the purpose of the grid, will choose a different way to control.

\subsubsection{PQ control strategy}

Constant power control strategy (PQ control strategy) is given by the given output power, in order to achieve the output side of the AC active power and reactive power control of a strategy.

PQ control strategy typical block diagram as shown below:

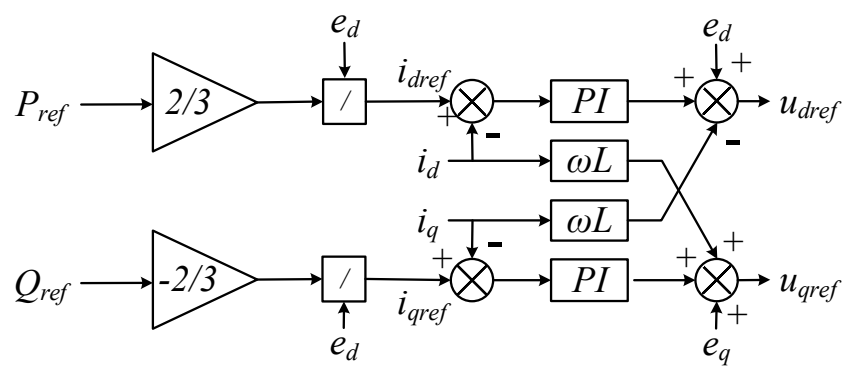

Figure 4 PQ control strategy typical block diagram. 
As shown in Fig. 4, the constant power control strategy is such that the power expectation values $P_{\text {ref }}$ and $Q_{\text {ref }}$ are converted into current reference values $i_{\text {dref }}$ and $i_{\text {qref }}$, and $i_{\text {dref }}$ and $i_{\text {qref }}$ are output as voltage reference values $u_{d \text { ref }}$ and $u_{q \text { ref }}$ by the PI regulator, so that the reference value Modulation, the inverter in the power of the electronic switch duty cycle to control, and ultimately the converter output of the ideal active power and reactive power.

\subsubsection{V / f control strategy}

Constant voltage constant frequency control strategy ( $\mathrm{V} / \mathrm{f}$ control strategy) is given by the given output voltage $U_{\text {ref }}$, and the expected output frequency $f_{\text {ref }}$, through the voltage outer ring and the current inner ring control, after three times the PI regulator control to achieve the output side of the output voltage and frequency control of a strategy.

V / f control strategy typical block diagram as shown below:

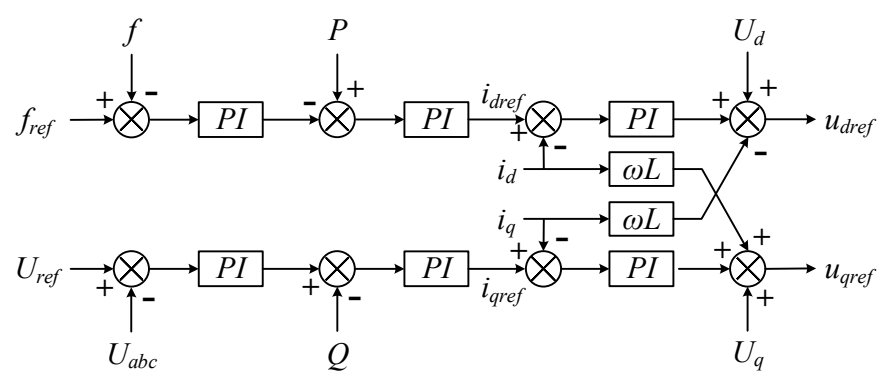

Figure $5 \mathrm{~V} / \mathrm{f}$ control strategy typical block diagram.

As shown in Figure 5, the voltage outer loop of the constant voltage constant frequency control strategy converts the output voltage expected value $U_{\text {ref }}$ and the output frequency expected value $f_{\text {ref }}$ into two current reference values $i_{\text {dref }}$ and $i_{\text {qref }}$ through the two PI links, and the current inner loop passes the PI regulator to convert $i_{d r e f}$ and $i_{\text {qref }}$ is the voltage reference value $u_{d r e f}$ and $u_{q r e f}$, and the reference value is controlled by PWM to control the duty cycle of each power electronic switch in the inverter, and finally the converter outputs the ideal voltage and frequency.

\subsubsection{Droop control strategy}

The droop control strategy (Droop control strategy) is a strategy for controlling the output voltage and frequency on the output side by a given frequency reference, voltage reference, and actual reference frequency, voltage, and power output.

Droop control strategy typical block diagram as shown below:

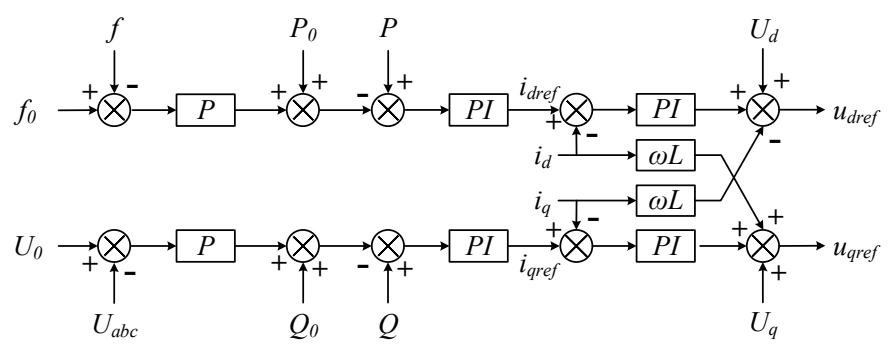

Figure 6 Droop control strategy typical block diagram.

As shown in Figure 6, the outer ring droop control strategy is the active power $\mathrm{P}$ and reactive power Q and voltage reference value $U_{0}$ and $f_{0}$ respectively with the frequency reference value of 
active power through the $\mathrm{P}$ ring after the reference value $P_{0}$ and reactive power reference value $Q_{0}$ the difference after subtracting the reference value of active power and reactive power $P_{0}$ power reference value $Q_{0}$, through the PI link into the current reference values of $i_{\text {dref }}$ and $i_{\text {qref }}$, the current loop PI regulator $i_{d r e f}$ and $i_{\text {qref }}$ output voltage reference value $u_{d \text { ref }}$ and $u_{\text {qref }}$, the reference value through the PWM modulation, the power electronic switch duty cycle control in converter, finally realize commutation the ideal output voltage and frequency.

\subsubsection{DC voltage control strategy}

The DC voltage control strategy is controlled by the voltage regulator and the current loop through a given regulator output voltage, and the desired q-axis output current (known as 0 ), through the PI regulator to achieve the DC side Output voltage to control a strategy.

DC voltage control strategy typical block diagram as shown below:

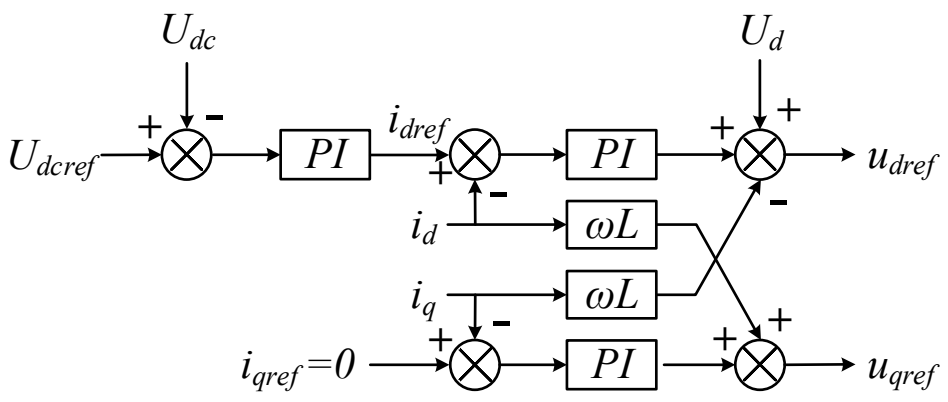

Figure 7 DC voltage control strategy typical block diagram.

As shown in Fig. 7, the voltage outer loop of the DC voltage control strategy converts the DC side output voltage expected value $U_{\text {dcref }}$ into the current reference value $i_{\text {dref }}$ through the PI link, and the resulting $i_{d r e f}$ and $i_{\text {qref }}$ are output as the voltage reference values $u_{d \text { ref }}$ and $u_{\text {qref }}$, with this reference value through the PWM modulation, the inverter in the power of the electronic switch duty cycle to control, and ultimately the converter output the ideal DC voltage.

\section{Multi - time scale modeling of AC / DC hybrid distribution network model}

\subsection{Model classification}

The converter and its control strategy model based on the mathematical model of the unit, according to the different input source time scale AC / DC hybrid power network model will be divided into two categories: AC / DC hybrid distribution network fixed value source model and AC / DC hybrid distribution network variable source model.

\subsection{AC / DC hybrid distribution network fixed value source model}

During the stable operation of AC / DC hybrid distribution network, the input terminal is stable, the visual input is a fixed value source, and the detailed model of input terminal can be ignored. Rectification and inverter status model diagram shown in Figure 8 and Figure 9, respectively: 


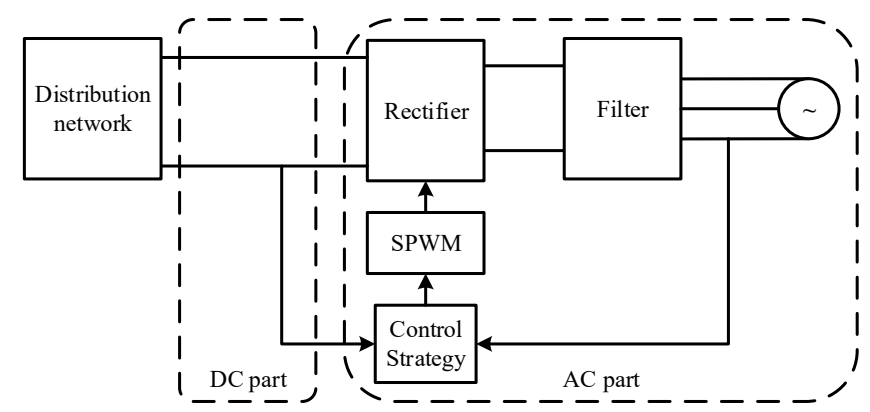

Figure 8 Schematic diagram of the steady state fixed value source model.

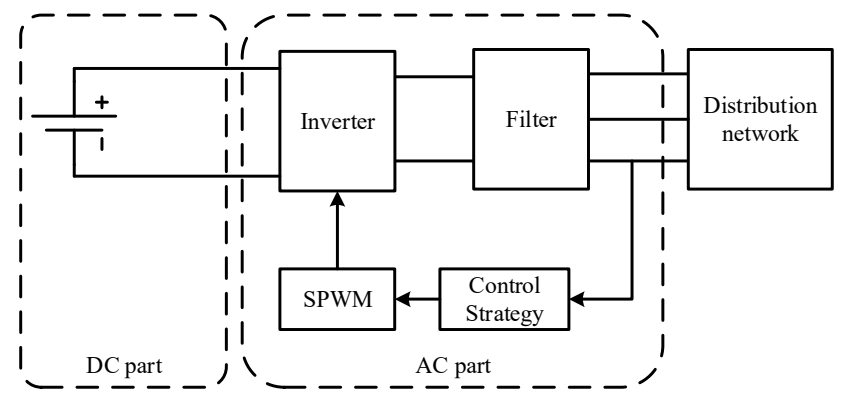

Figure 9 Schematic diagram of the inverter state fixed value source model.

In Figure 8, the steady-state model of the rectifier is controlled by the rectifier control strategy, which can realize the simulation of the AC side DC side power supply of AC / DC hybrid power distribution network.

In Figure 9, the inverter state fixed value source model is used to control the inverter through the inverter control strategy, which can realize the simulation of the DC side AC side power supply of $\mathrm{AC} / \mathrm{DC}$ hybrid power distribution network.

\subsection{AC / DC hybrid distribution network variable source model}

In the AC / DC hybrid distribution network inverter, if the consideration of the DC side of the external conditions (such as temperature variables, variables in light intensity, wind power, etc.) on the impact of distributed power plant output, AC / DC inverter needs to establish the state of distribution network variable source model with a detailed model of distributed power generation equipment. Taking the photovoltaic power generation unit as an example, AC / DC hybrid distribution network variation source diagram of the inverter model shown in Figure 10.

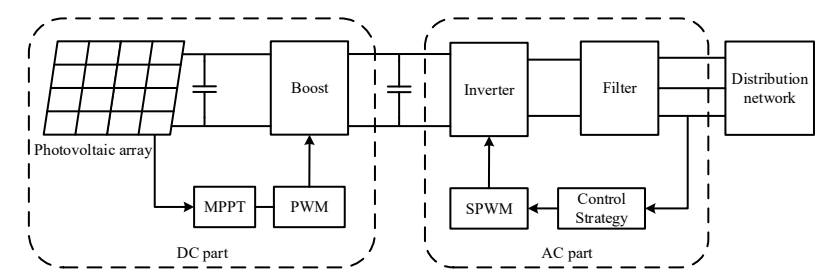

Figure 10 Schematic diagram of the inverter state variable source model. 


\section{Simulation Analysis of Multi - time Scale Model for AC / DC Hybrid Distribution}

Network

\subsection{Application scenario}

For comparing the two different scales of $\mathrm{AC} / \mathrm{DC}$ hybrid power network model simulation performance, the two different time scales of AC / DC hybrid power network model to access the IEEE33 node example, which variables the DC part of variable source model selection model of PV generation model.

As shown in Fig. 11, the AC / DC hybrid distribution network model is connected to the MV distribution network via the transformer. The access point is the end node (node 17) of the longest feeder in the example. Among them, the variable source model DC side of the distributed power generation unit using photovoltaic power generation unit, rated voltage of $380 \mathrm{~V}$, rated output power of $50 \mathrm{~kW}$, reactive power of 0 .

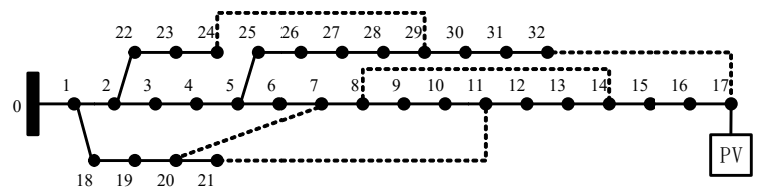

Figure 11 Topology of Photovoltaic Application Scenarios.

In this paper, the simulation performance of AC / DC hybrid distribution network model at different scales is based on the above comparison. Based on the limitation of space, this paper only carries on the simulation and analysis of the AC and DC hybrid distribution network with the variable source inverter model and the variable source inverter model with the photovoltaic power source as the distributed power source. Simulation of the hardware environment for the installation of MATLAB R2014a PC, which CPU clocked at 1.8GHz, 8GB memory.

In this paper, two different scales of AC / DC hybrid distribution network model, under the same hardware conditions, the simulation accuracy of the model comparison test. The simulation results show that the simulated output power waveform is compared with the Simulink oscilloscope, and the accuracy of the AC / DC hybrid distribution network simulation at different time scales is compared and analyzed. The simulation model is shown in Figure 12.

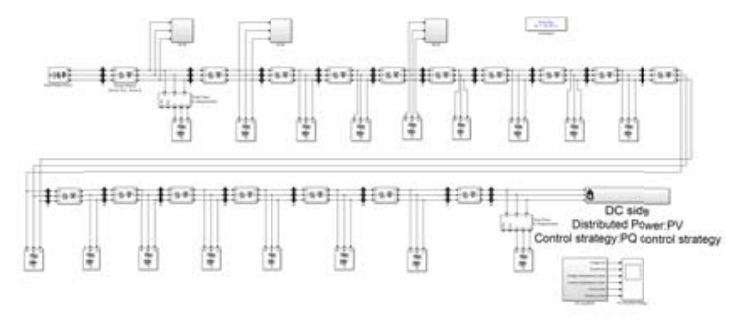

Figure 12 Multi-scale Simulation Model of Grid-connected Photovoltaic Power Generation System.

\subsection{Simulation result}

Based on the analysis of the previous section, in order to compare the output power of different scale models of AC / DC hybrid distribution network under the same conditions, the expected active power of the output side of the converter is set to $5 \times 10^{4} \mathrm{~W}$, and the simulation step is $5 \mu \mathrm{s}$. The model is measured in stable operation, measuring its output power: 


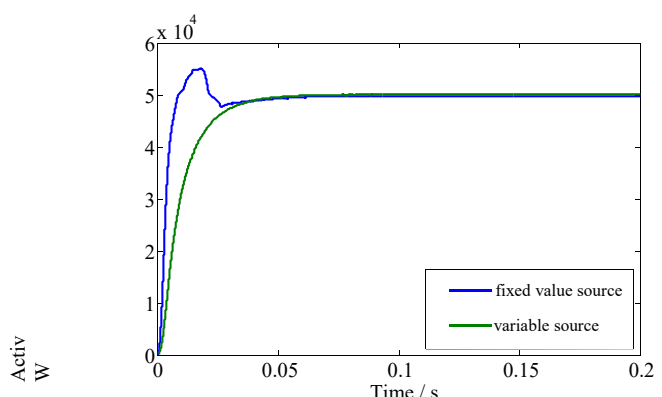

Figure 13 Different scale distribution network model converter output side active power.

As shown in Figure 13, the oscilloscope waveform display of two different scales of AC / DC hybrid distribution network model converter output side of the active power can be stable within $0.04 \mathrm{~s}$ within the rated power expected.

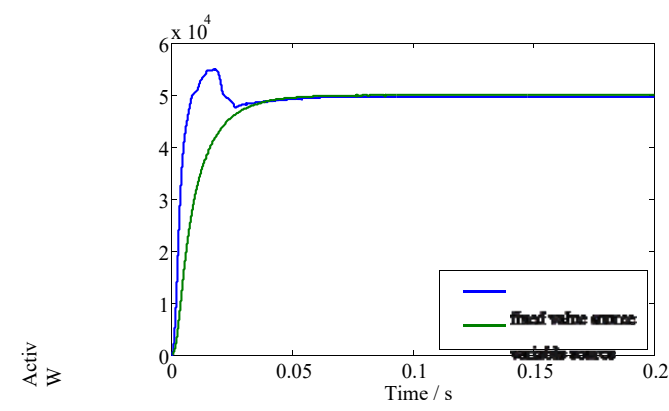

Figure 14 Different scale mating network model converter output side stable active power.

As shown in Figure 14, after the 0.04s system runs to steady state, the oscilloscope displays the waveform, the value of the source model because it contains power electronic switching devices and control strategy model, and capacitive inductance and other energy storage components, the output power slightly fluctuates, and the average output power is less than the rated power. The variable source model also has the slight fluctuation of the output active power of the variable source model due to the inclusion of complex nonlinear elements, and the output power of the converter model is consistent with the expected output power at the same time. From the above analysis, it can be seen that the simulation accuracy of AC / DC hybrid distribution network source model is higher than that of fixed value source model.

\section{Conclusion}

The multi-scale modeling work is carried out for the AC / DC hybrid distribution network, and through the comparison of the simulation accuracy of different scale models, the conclusion can be summarized as follows: The simulation accuracy of the output power of the converter is better than that of the fixed source model.

In summary, in the AC-DC hybrid distribution network simulation process, when the hardware condition of the PC is high, the variable source model should be selected to simulate. When the hardware condition of the PC is low, the fixed value source model can be selected for simulation.

\section{Acknowledgements}

This work was supported by Project of Electric Power Research Institute of State Grid NingXia Electric Power Company (PDB11201601764). 


\section{References}

[1] ZHENG Chao, ZHOU Xiao-xin, LI Xuo-mei, et al. Dynamic modeling and transient simulation for voltage source converter based HVDC [J]. Power System Technology, 2005, (16): 1-5.

[2] ZHENG Chao, etc. Mathematical modeling and simulation analysis for voltage source converter based HVDC [D]. Beijing: China Electric Power Research Institute, 2006.

[3] LU Xiao-jun, AN Ting, WU Ya-nan, LI Yun-feng, WEN Jin-yu, et al. Analytical Modeling and Verification of a 2-level Voltage Source Converter [J]. Smart Grid, 2015, (05): 371-380.

[4] WANG He, LI Guo-qing, et al. Control strategy of microgrid with different DG types [J]. Electric Power Automation Equipment. 2012, (05): 19-23.

[5] WANG Cheng-shan, GAO Fei, LI Peng, HUANG Bi-bin, DING Cheng-di, YU Hao. Control Strategy Research on Low Voltage Microgrid [J]. Proceedings of the CSEE, 2012, (25): 2-9.

[6] WANG Cheng-shan, LI Yan, PENG Ke, et al. Overview of Typical Control Methods for Grid-Connected Inverters of Distributed Generation [J]. Proceedings of the CSU-EPSA, 2012, (02): 12-20.

[7] LV Zhi-peng, LUO An, RONG Fei, GUO Lu, et al. Micro-grid PQ Control Strategy Analysis Under Utility Voltage Imbalance [J]. Power Electronics, 2010, (06): 71-74.

[8] CHEN Zhi-bao, DING Jie, ZHOU Hai, et al. Voltage power super-short-term prediction model of ground-based cloud image combined with radial basis function artificial neural network [J]. Proceeding of the CSEE, 2015, 03: 561-567.

[9] YOU Guo-dong, LI Ji-sheng, HOU Yong et al. Modeling and MPPT control of partially shielded photovoltaic power generation system [J]. Power System Technology, 2013, 11: 3037-3045.

[10] SUN Ying-yun, HOU Jian-lan, LI Run, et al. Photovoltaic array model based on implicit complementary problem and its algorithm [J]. Proceeding of the CSEE, 2014, 34: 6066-6073. 